\title{
Improvement of enzymatic hydrolysis of rice straw by $\mathbf{N}$ - methylmorpholine-N-oxide (NMMO) pretreatment
}

\author{
Nafiseh Poornejad ${ }^{1}$, S.M. Amin Salehi ${ }^{1,{ }^{*}}$, Keikhosro Karimi ${ }^{1}$, M.J. Taherzadeh ${ }^{2}$, Tayebeh \\ Behzad $^{1}$ \\ ${ }^{1}$ Department of Chemical Engineering, Isfahan University of Technology, Isfahan, Iran \\ ${ }^{2}$ School of Engineering, University of Borås, Borås, Sweden \\ *Corresponding author.E-mail: a.salehi@ce.iut.ac.ir
}

\begin{abstract}
Food versus energy" analysis resulted in demanding raw materials which don't have conflict with food industries. The lignocellulosic materials are the most interested option, since not only these materials don't have conflict with food industries, but also there several economical and environmental advantages in those substrates for bioethanol production.

However, the lignocellulosic materials are recalcitrant to both acid and enzymatic hydrolysis. As a result, some pretreatment steps must be taken before hydrolysis. One of the most effective pretreatment methods is treatment with cellulosic solvent.

In this work rice straw, an agricultural residue which is mainly unused was pretreated with an industrial solvent, $\mathrm{N}$-methylmorpholine-N-oxide (NMMO). The pretreatments were performed at $120^{\circ} \mathrm{C}$ for $1,3,5$ hours with $85 \%$ NMMO solution. The treated materials were then subjected to enzymatic hydrolysis by a mixture of commercial cellulase and glucosidase.

The results showed significant improvement on the enzymatic hydrolysis. Almost complete hydrolysis of the cellulose in the straw was observed after $5 \mathrm{~h}$ treatment with $\mathrm{NMMO}$ at ambient pressure and $120^{\circ} \mathrm{C}$.
\end{abstract}

Keywords: pretreatment, $N M M O$, rice straw, enzymatic hydrolysis

\section{Introduction}

Nowadays different resources are applied for ethanol production in all over the world. The dominant of these resources are agricultural residue such as corn, sugar, starch, and lignocellulosic materials. Increasing growth of industrial ethanol production cause debates on "food \& fuel" and afflicted the food industries [1]. Therefore, researchers are trying to find renewable resources that don't have conflict with food resources. Cellulosic wastes are the most possible option. The largest source for this purpose is lignocellulosic materials such as agricultural residue (bagasse, sugar cane stalk etc), forestry wastes (hard and soft wood), and municipal materials.

For a long time, researchers are aspiring to enhance digestibility of lignocellulosic biomass to convert cellulose to ethanol [2]. These materials are reluctant to enzymatic hydrolysis, as a result, conversion of them to ethanol consists of five main steps as shown Fig. 1:

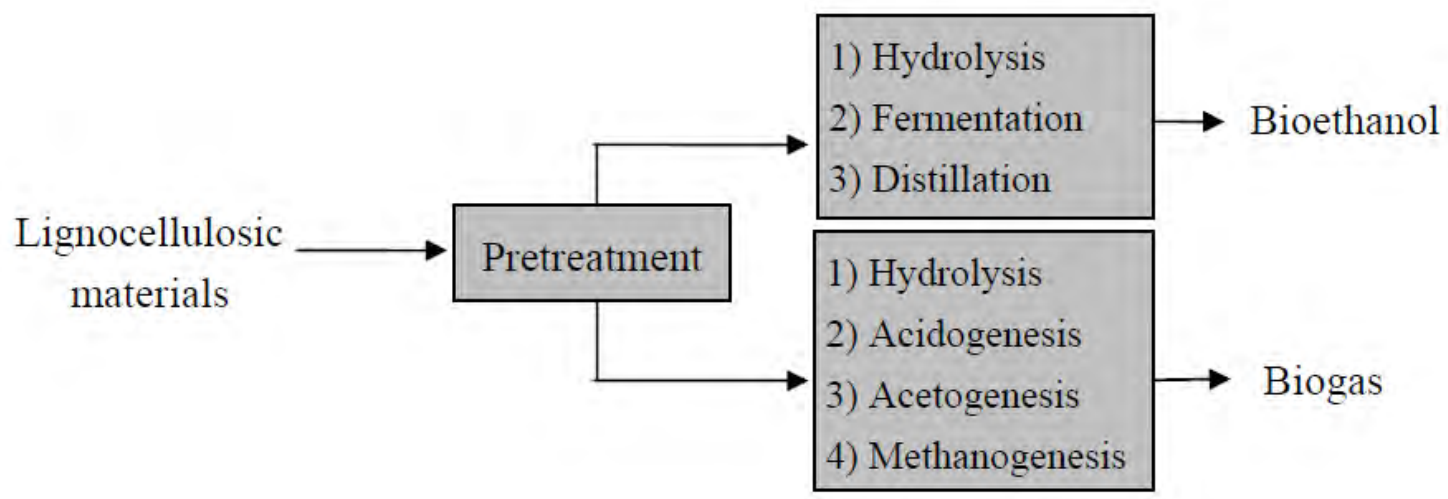

Fig. 1. Pretreatment of lignocellulosic materials prior to bioethanol prodyction [3] 
Lignocellulosic materials consist of mainly three different types of polymers, namely cellulose, hemicelluloses, and lignin which are associated with each other [2].

Rice straw is one of the lignocellulosic waste materials could be found in the world. The estimation of annual production of rice by FAO is about 600 million tons per year in 2004 . On the other hand, every kilogram of grain harvested is associated with production of $1-1.5 \mathrm{~kg}$ of the straw. It gives an estimation of global production of 600-900 million tons per year of straw. The ways of the disposition of rice straw are limited by the great bulk of material, slow degradation in the soil, harboring of rice stem diseases, and high mineral content. Field burning is the major practice for getting rid rice straw, but it increases air pollution and consequently affects the public health. Many counties in Western Europe have already banned open field burning and some other countries have considered it seriously [4].

Depend on the structure of lignicellulosic materials (the portion of these three polymers), the most effective pretreatment method could be selected. There are different kinds of pretreatment. The main categories are: physical pretreatment, chemical and physicochemical pretreatment, and biological pretreatment [3].

One of the chemical pretreatment methods is using solvent for solving the substrate and then regenerating the cellulose part by adding an anti solvent. N-methylmorpholine-N-oxide (NMMO) is among the nonderivatizing solvents which can dissolve cellulose by breaking the intermolecular forces. NMMO is one of the direct solvent for cellulose, which is nowadays applied in the industrial Layocell process. This process is one of the modern and environmentally friendly industrial fiber-making technologies with direct dissolution of cellulose without chemical derivatization. $\mathrm{T}$ he solvent does not produce toxic waste pollutants, and can be recycled with over 98\% recovery. After dissolution in NMMO, cellulose can be regenerated by rapid precipitation with and anti solvent, which is usually water. The dissolution in NMMO can change the crystal structure of cellulose.

The treatment with NMMO has several advantages in comparison with other pretreatment methods such as conventional acid, alkali, and thermal pretreatments. Most of the other pretreatment methods change the composition of the substrates by e.g., removing hemicelluloses by dilute-acid or lignin by sodium hydroxide. However, NMMO keep the same composition as is reported in many researches. The NMMO process is performed in milder conditions, such as atmospheric pressure and lower temperature $\left(<130^{\circ} \mathrm{C}\right)$ than for e.g., in dilute-acid, which results in less energy consumption of the entire process. In addition, no need for chemical neutralization and the possibility of more than $98 \%$ recovery of the solvent in industrial processes are other significant property of using NMMO. These properties in addition to the high amount of conversion of cellulose to ethanol which is presented here make the pretreatment with NMMO a good alternative for lignocellulosic ethanol production [5].

Different researchers have been working on the effect of NMMO on enzymatic hydrolysis of various substrates. As reported by Chai-Hung et al, the amount of reducing sugar released from sugar cane bagasse after $7 \mathrm{hr}$ pretreatment in $100^{\circ} \mathrm{C}$ by NMMO was at least two times more than untreated sugar cane bagasse [6]. In another study, Shafiei et al have reported an increase about $75 \%$ and $50 \%$ in the yield of enzymatic hydrolysis of spruce and oak respectively after $3 \mathrm{hr}$ NMMO pretreatment in $130^{\circ} \mathrm{C}$ in comparison with untreated substrates $[5]$. 
Because of all the mentioned reasons, the current study aimed at pretreatment of rice straw with NMMO.

\section{Methodology}

\subsection{Raw materials and their analysis}

The rice straw used in these experiments was obtained from Lenjan field (Isfahan, Iran). The original length was between 2 and $50 \mathrm{~mm}$. It was partly screened to achieve a size of less than $0.8 \mathrm{~mm}$ prior to the pretreatment. Its dry weight content was measured at $105^{\circ} \mathrm{C}$ for $24 \mathrm{~h}$. The rice straw structure is reported by Karimi et al [4].

\subsection{Pretreatment}

The $85 \%$ NMMO solution was used for the pretreatment. The pretreatment performed in $200 \mathrm{ml}$ Erlenmeyer flasks, where $1 \mathrm{~g}$ of rice straw (dry weight) was added to $19 \mathrm{~g}$ of the NMMO solvent and mixed every $15 \mathrm{~min}$. Treatment was done in an oil bath at $120^{\circ} \mathrm{C}$ and for three different durations (1, 3 , and $5 \mathrm{~h}$ ). The pretreated rice straw was then regenerated by addition of $30 \mathrm{ml}$ boiling deionized water, followed by vacuum filtration and washing until a clear filtrate appeared.

\subsection{Enzymatic hydrolysis}

NMMO-treated and untreated rice straw was subjected to $72 \mathrm{~h}$ enzymatic hydrolysis using $20 \mathrm{FPU}$ cellulase and 30 IU $\beta$-glucosidase per gram cellulose [7]. Then the reminder solid was separated by centrifuge from the supernatant. The yield of enzymatic hydrolysis is defined as (grams of glucose released + grams of xylose released)/(grams of initial solid used for hydrolysis $\times 1.111)$. The dehydration factor (1.111) is used to convert cellulose chains to glucose monomers.

\subsection{Analytical method}

All the samples were analyzed by high-performance liquid chromatography (HPLC), equipped with UV/VIS and RI detectors (Jasco International Co., Tokyo, Japan). Glucose and xylose were determined by an Aminex HPX-87P column (Bio-Rad, Richmond, CA, USA) at $80^{\circ} \mathrm{C}$. Deionized water was used as efluent at a flow rate of $0.6 \mathrm{ml} / \mathrm{min}$.

\section{Result and discussion}

As shown in Fig.2, $3 \mathrm{~h} \mathrm{~N}$ MMO-treated substrate shows significant improvement in hydrolysis than $1 \mathrm{~h} \mathrm{~N}$ MMO-treated substrate and there is no s ignificant change in the performance of NMMO after $5 \mathrm{~h}$ pretreatment in comparison with $3 \mathrm{~h}$ pretreatment. The glucose concentration in hydrolysate (liquid phase) after $24 \mathrm{~h}$ increase significantly by pretreatment, as a result, the rate of hydrolysis improve radically. The rate of hydrolysis (concentration after $24 \mathrm{~h}$ ) increases about $100 \%$ after even $1 \mathrm{~h} \mathrm{t}$ reated straw in comparison with untreated straw and the concentration of glucose increases about $400 \%$. All of these show significant improvement in enzymatic digestibility of rice straw after being treated with NMMO. That's because of destruction of intermolecular interactions. 


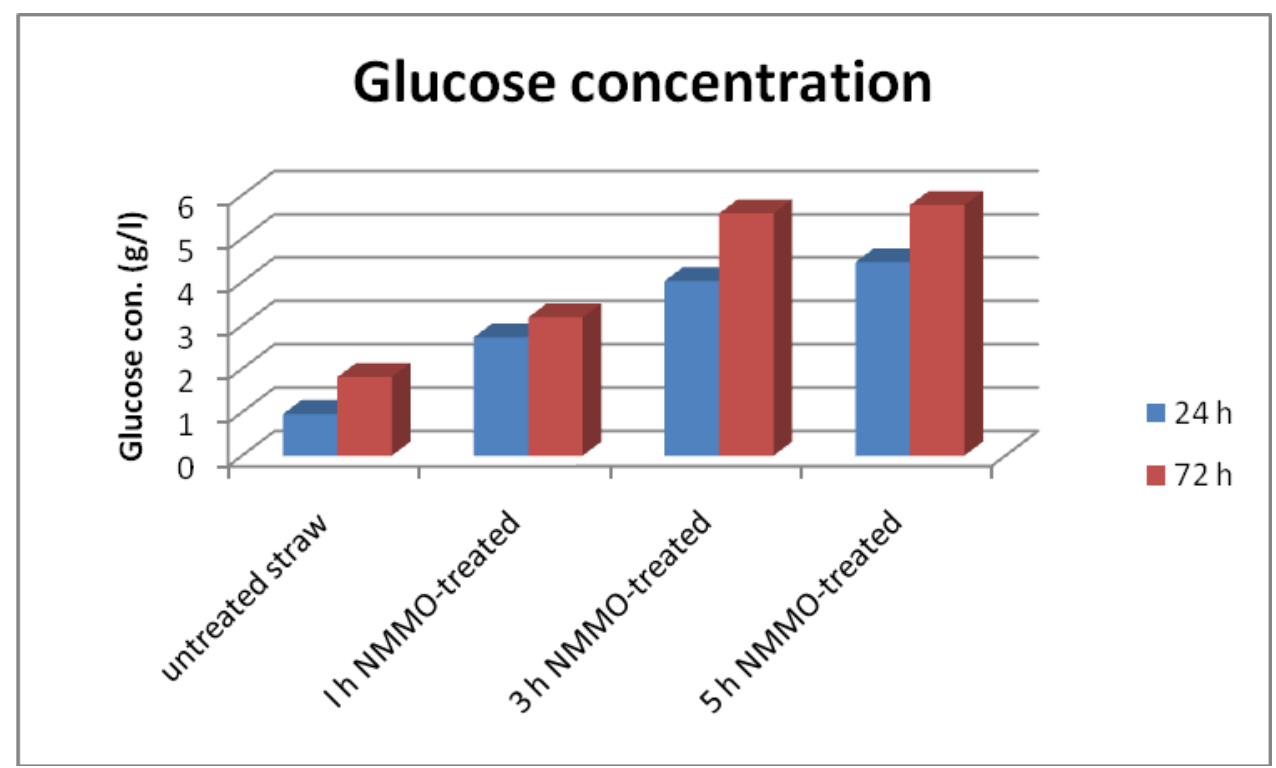

Fig. 2. Glucose concentration in hydrolysate during enzymatic hydrolysis

As shown in Fig. 3, x ylose concentration increases, too. The xylose concentration by $5 \mathrm{~h}$ treatment increases about $500 \%$ after $72 \mathrm{~h} \mathrm{~h}$ ydrolysis, moreover, the rate of hydrolysis increases by $300 \%$. These results show undeniable superiority of NMMO over other solvents.

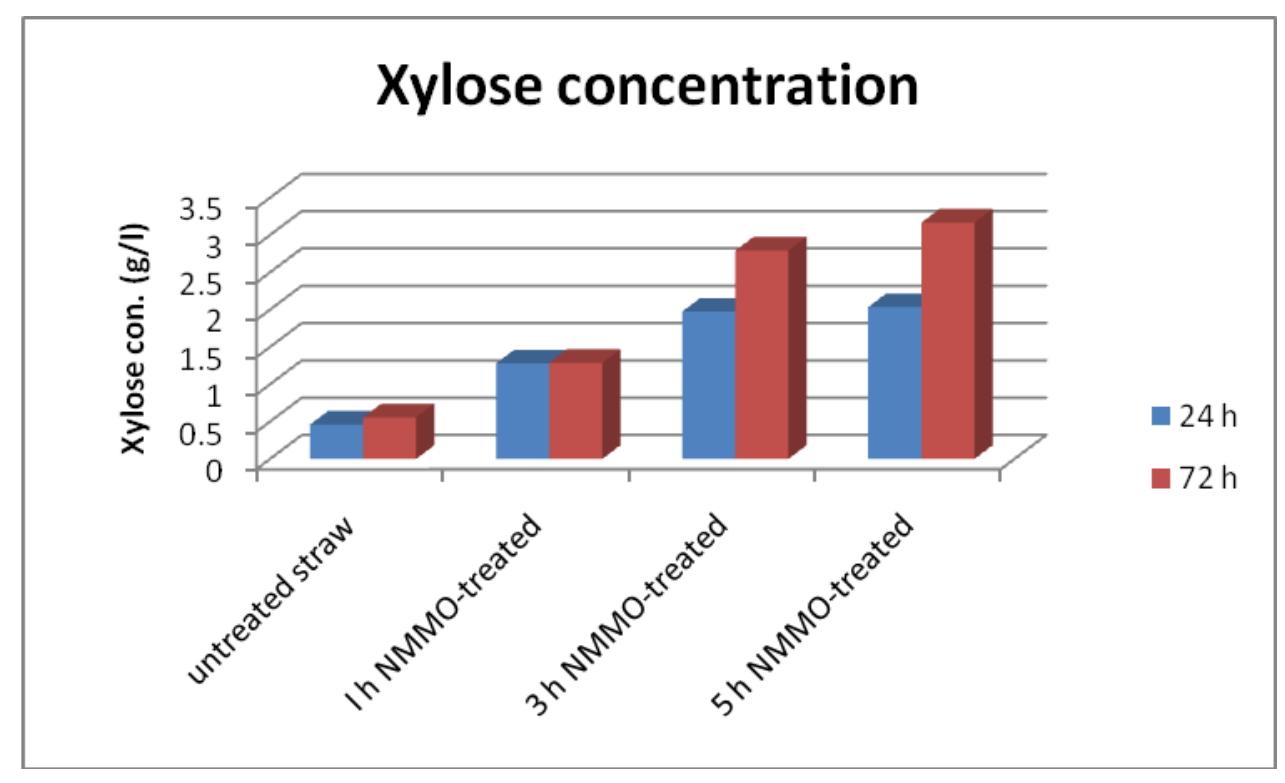

Fig. 3. Xylose concentration in hydrolysate during enzymatic hydrolysis

As shown in the last figure (Fig. 4.), the total sugar yield increases from $23.69 \%$ (untreated straw) to $84.42 \%$ ( $5 \mathrm{~h} \mathrm{NMMO-treated} \mathrm{straw).} \mathrm{This} \mathrm{means} \mathrm{an} \mathrm{outstanding} \mathrm{improvement} \mathrm{in}$ terms of industrial process. 


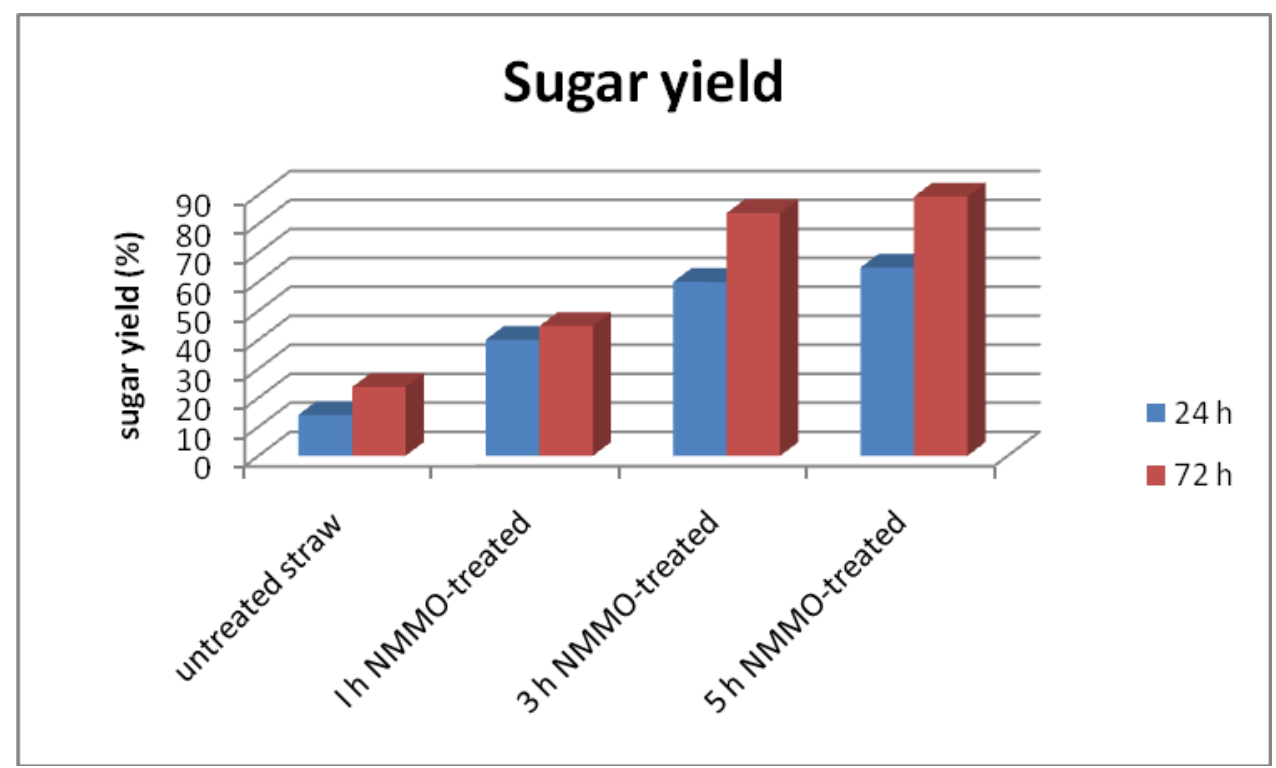

Fig. 4. Total yield of sugar during hydrolysis

\section{Conclusion}

Treatment with NMMO would be one of the promising ways for preparing rice straw for ethanol production. Having the capacity of being recovered and then reused, NMMO is a good alternative solvent for cellulose. As discussed above in atmospheric pressure and $120^{\circ} \mathrm{C}$ after $5 \mathrm{hr}$ pretreatment the yield of about $90 \%$ could be achieved after $72 \mathrm{hr}$ enzymatic hydrolysis of rice straw.

\section{References}

[1] G.J. Hayes, An examination of biorefining processes, catalysts and challenges, Catal. Today (2008), doi:10.1016/j.cattod.2008.04.017.

[2] A.T.W.M. Hendriks, G. Zeema, pretreatment to enhance the digestibility of lignocellulosic biomass, Bioresource Technology 100 (2009) 10-18.

[3] M.J. Taherzadeh, K. Karimi, pretreatment of lignocellulosic wastes to improve Ethanol and Biogas production: A review, Int. J. Mol. Sci. 2008, 9, 1621-1651; DOI: 10.3390/ijms9091621.

[4] K. Karimi, Sh. Kheradmandinia, M.J. Taherzadeh, Conversion of rice straw to sugars by dilute acid hydrolysis, Biomass and Bioenergy 30(2006)247-253.

[5] M. Shafiei, K. Karimi, M.J. Taherzadeh, Pretreatment of spruce and oak by Nmethylmorpholine-N-oxide (NMMO) for efficient conversion of their cellulose to ethanol, Bioresource Technology 101 (2010) 4914-4918.

[6] Ch-H. Kuo, Ch-K. Lee, Enhanced enzymatic hydrolysis of sugar cane bagasse by Nmethylmorpholine-N-oxide pretreatment, Bioresource Technology 100(2009) 866-871.

[7] A. Jeihanipour, M.J. Taherzadeh, Ethanol production from cotton based waste textiles, Bioresource Technol 100(2):1007-1010. 